\title{
Dynamic Output Feedback Robust MPC with Input Saturation Based on Zonotopic Set-Membership Estimation
}

\author{
Xubin Ping, ${ }^{1}$ Bo Qian, ${ }^{1}$ and Ning Sun ${ }^{2}$ \\ ${ }^{1}$ School of Electro-Mechanical Engineering, Xidian University, Xian 710071, China \\ ${ }^{2}$ School of Electronic Engineering, Xidian University, Xian 710071, China \\ Correspondence should be addressed to Xubin Ping; xbping@xidian.edu.cn
}

Received 11 November 2015; Accepted 22 March 2016

Academic Editor: Dane Quinn

Copyright (c) 2016 Xubin Ping et al. This is an open access article distributed under the Creative Commons Attribution License, which permits unrestricted use, distribution, and reproduction in any medium, provided the original work is properly cited.

\begin{abstract}
For quasi-linear parameter varying (quasi-LPV) systems with bounded disturbance, a synthesis approach of dynamic output feedback robust model predictive control (OFRMPC) with the consideration of input saturation is investigated. The saturated dynamic output feedback controller is represented by a convex hull involving the actual dynamic output controller and an introduced auxiliary controller. By taking both the actual output feedback controller and the auxiliary controller with a parameterdependent form, the main optimization problem can be formulated as convex optimization. The consideration of input saturation in the main optimization problem reduces the conservatism of dynamic output feedback controller design. The estimation error set and bounded disturbance are represented by zonotopes and refreshed by zonotopic set-membership estimation. Compared with the previous results, the proposed algorithm can not only guarantee the recursive feasibility of the optimization problem, but also improve the control performance at the cost of higher computational burden. A nonlinear continuous stirred tank reactor (CSTR) example is given to illustrate the effectiveness of the approach.
\end{abstract}

\section{Introduction}

In control system design, input saturation nonlinearity can severely degrade closed-loop system control performance and may lead to the instability of a closed-loop system $[1,2]$. Hence, stability analysis of controller design with the consideration of input saturation has received extensive attention. An approach to deal with input saturation is to penalize the control input such that input constraint is never violated. This approach is common in the synthesis approach of MPC; for example, see [3-9]. In the synthesis approach of MPC, at each sampling time, the on-line optimization problem is solved to obtain an optimal controller, which considers physical constraints (e.g., input constraint and output constraint) and stability condition. Here, we refer to MPC with guaranteed stability as the synthesis approach, which is usually based on recursive feasibility; that is, the optimization problem is feasible for all time if it is feasible at the initial time $[8,10]$.
Linear parameter varying (LPV) systems offer a promising framework for modeling and control of a large class of nonlinear systems [11]. By applying plenty of methods and techniques developed for linear systems, LPV models provide efficient ways to deal with some complex nonlinear systems [12-14]. When the scheduling parameters of LPV systems are exactly known at the current time but unknown in future, it is quasi-LPV system [7-9]. In robust MPC (RMPC) studies, a real nonlinear system is often approximated or included by polytopic uncertainty and then represented by LPV description. The synthesis approach of RMPC is often solved by on-line min-max optimization, which considers all the possible realizations of polytopic model parametric uncertainty, constraints, and/or not bounded disturbance; for example, see [3, 5, 7-9]. For the control of nonlinear systems subject to uncertainty, constraints, and faults in the control actuators, the authors in $[15,16]$ investigate a RMPC design for fault-tolerant control. Furthermore, when true states are unmeasurable, an output feedback MPC controller based 
on state observer is also considered in [16]. The feedback controller design in the synthesis of RMPC is often enforced by stringent constraints, which is usually conservative for the optimized controller. To reduce the conservatism of the controller design, the authors in [17] take a parameter-dependent controller, which improves the control performance in [3]. Free control moves (the control moves are the decision variables in on-line optimization problem) are introduced in [4-6] for state feedback controller design, which can also improve the control performance in [3]. Furthermore, the time-varying parameters of LPV systems having bounds on their rate of variation are considered in $[5,6]$, which further reduce the conservatism of the controller design. By introducing one free control move, the authors in [18] generalize the procedure in [4] to the case when the state is unmeasurable.

In [7-9], the dynamic OFRMPC approach considers quasi-LPV systems with bounded disturbance. By taking controller parameters with a parameter-dependent form, the main optimization problem is solved as convex optimization. The estimation error sets in [7-9] are represented by polyhedral sets (in [9], the estimation error set is represented by a zonotope, which is a special case of polyhedral set). In $[7,9]$, at each sampling time, the auxiliary optimization problem can not only refresh the bounds of estimation error set, but also determine whether to solve the main optimization at the next sampling time or not. However, the iterative auxiliary optimization problem in [7] increases the on-line computational burden, and the recursive feasibility of the optimization problem in $[7,9]$ is not guaranteed. The algorithm in [8] can guarantee the recursive feasibility of the optimization problem, while the control performance is worse than that in [9]. The estimation error set in [9] is refreshed by zonotopic set-membership estimation, which improves the control performance in $[7,8]$. Furthermore, in [7-9], an ellipsoidal bounded disturbance set is considered in the main optimization problem. However, an outer approximation polyhedral set of the ellipsoidal bounded disturbance is utilized in the process of refreshing estimation error set, which may introduce conservatism.

To reduce the conservatism of OFRMPC controller design in [7-9] and then improve the control performance, the present paper considers a synthesis approach of dynamic OFRMPC with input saturation for the quasi-LPV system with bounded disturbance. Different from $[15,16]$, we assume that the system is under nominal operation and control actuator faults are not considered. The saturated dynamic output feedback controller is expressed by a convex hull involving the parameter-dependent form of both the actual dynamic output feedback controller and the auxiliary feedback controller, which is different from the saturated output feedback controller in $[1,19,20]$. The parameterdependent form of controller can help to formulate the main optimization problem as convex optimization. Then the main optimization problem can be solved by a linear matrix inequality (LMI) tool. In the main optimization problem, only the constraints on the auxiliary feedback controller are considered, which introduce more optimization freedom for the the actual dynamic output feedback controller. By this way, the conservatism of output feedback controller design is reduced such that the control performance can be improved. In the process of refreshing estimation error set, based on the estimation error equation, the polyhedral estimation error set is refreshed by zonotopic set-membership estimation. Then the obtained polyhedral estimation error set compares with the ellipsoidal estimation error set obtained from the invariance condition of the augmented closed-loop system. By properly refreshing the bounds of estimation error set, it not only obtains the bounds of polyhedral estimation error set, but also guarantees the recursive feasibility of the optimization problem. Furthermore, different from [7-9], both the main optimization problem and the refreshment of estimation error set consider componentwise bounded disturbance, which is confined by a polyhedral set and also can be represented by a zonotope. Hence, the outer approximation of bounded disturbance in the process of refreshing estimation error set is not involved. Compared with $[8,9]$, the consideration of input saturation in the main optimization problem improves the control performance at the cost of higher on-line computational burden.

Notations. For any vector $x$ and positive-definite matrix $W$, $\|x\|_{W}^{2} \triangleq x^{\mathrm{T}} W x \cdot x(i \mid k)$ is the value of $x$ at time $k+i$, predicted at time $k . I$ is the identity matrix with appropriate dimension. $\varepsilon_{M} \triangleq\left\{\xi \mid \xi^{\mathrm{T}} M \xi \leq 1\right\}$ denotes the ellipsoid associated with the symmetric positive-definite matrix $M$. All vector inequalities are interpreted in a componentwise sense. An element belonging to $\operatorname{Co}\{\cdot\}$ means that it is a convex combination of the elements in $\{\cdot\}$, with the scalar combing coefficients being nonnegative and their sum is equal to 1 . The symbol " $\star$ " induces a symmetric structure in the matrix inequalities. A value with superscript “*” means that it is the optimal solution of the optimization problem. The time dependence $(k)$ of the MPC decision variables is often omitted for brevity.

Let an interval $[a ; b]$ be defined as the set $\{x: a \leq$ $x \leq b\}$. The unitary interval is $\mathbf{B}=[-1 ; 1]$. A box $\left(\left[a_{1} ; b_{1}\right], \ldots,\left[a_{n} ; b_{n}\right]\right)^{\mathrm{T}}$ is an interval vector. A unitary box in $\mathfrak{R}^{m}$, denoted by $\mathbf{B}^{m}$, is a box composed by $m$ unitary intervals. The Minkowski sum of two sets $X$ and $Y$ is defined by $X \oplus Y=$ $\{x+y: x \in X, y \in Y\}$.

Definition 1. An $m$-zonotope in $\Re^{n}$ can be defined as the linear image of an $m$-dimensional hypercube in $\mathfrak{R}^{n}$, where $m$ is the order of the zonotope and satisfies $m \geq n$. Given a vector $p \in \mathfrak{R}^{n}$ and a matrix $H \in \mathfrak{R}^{n \times m}$, an $m$-zonotope is the following set:

$$
Z \triangleq p \oplus H \mathbf{B}^{m}=\left\{x \in \mathfrak{R}^{n} \mid x=p+H z, z \in \mathbf{B}^{m}\right\}
$$

This is the Minkowski sum of the $m$-segments defined by $m$ columns of matrix $H$ in $\Re^{n}$. The center of zonotope $Z$ is vector $p ; H=\left[h_{1} \cdots h_{i} \cdots h_{m}\right]$, with $h_{i} \in \mathfrak{R}^{n}, i \in\{1, \ldots, m\}$, are the generators of zonotope $Z$. Zonotopes are the special case of polyhedral sets and can be represented by the convex combination of their vertices. 


\section{Problem Statement}

Consider the following discrete-time uncertain LPV system:

$$
\begin{aligned}
x(k+1) & =A(k) x(k)+B(k) \sigma[u(k)]+D(k) w(k), \\
y(k) & =C(k) x(k)+E(k) w(k),
\end{aligned}
$$

where $u \in \mathfrak{R}^{n_{u}}, x \in \mathfrak{R}^{n_{x}}, y \in \mathfrak{R}^{n_{y}}$, and $w \in \mathfrak{R}^{n_{w}}$ are the input, state, output, and disturbance, respectively. The disturbance is componentwise bounded satisfies $\left|w_{j}(k)\right| \leq$ $1, j \in\left\{1, \ldots, n_{w}\right\}$; that is, $w(k)$ is bounded in a polyhedral set, which can be represented by the following zonotope:

$$
w(k) \in \mathbb{D} \triangleq 0 \oplus \mathbf{B}^{n_{w}} .
$$

The output constraint is required to satisfy

$$
-\bar{\psi} \leq \Psi y(k+1) \leq \bar{\psi},
$$

where $\bar{\psi}=\left[\bar{\psi}_{1}, \bar{\psi}_{2}, \ldots, \bar{\psi}_{q}\right]^{\mathrm{T}}, \bar{\psi}_{j}>0, j \in\{1, \ldots, q\}$, and $\Psi \in \mathfrak{R}^{q \times n_{y}}$. The function $\sigma: \mathfrak{R}^{n_{u}} \rightarrow \mathfrak{R}^{n_{u}}$ is a vector valued standard saturation function; that is, $\sigma(u(i \mid k))=\left[\sigma\left(u_{1}(i \mid\right.\right.$ $\left.k)), \sigma\left(u_{2}(i \mid k)\right), \ldots, \sigma\left(u_{n_{u}}(i \mid k)\right)\right]$, and $\sigma\left(u_{r}(i \mid k)\right)=$ $\operatorname{sgn}\left[u_{r}(i \mid k)\right] \min \left\{1,\left|u_{r}(i \mid k)\right|\right\}, r \in\left\{1, \ldots, n_{u}\right\}$. Here, we have slightly abused the notation by using $\sigma$ to denote both the scalar valued and vector valued saturation functions. The time-varying system matrices $[A|B| C|D| E](k)$ are known to vary within a polytope $\Omega$; that is, $[A|B| C|D|$ $E](k) \in \Omega \triangleq \operatorname{Co}\left\{\left[A_{l}\left|B_{l}\right| C_{l}\left|D_{l}\right| E_{l}\right], l=\{1, \ldots, L\}\right\}$, and there exist nonnegative coefficients $\lambda_{l}(k)$ 's with $\sum_{l=1}^{L} \lambda_{l}(k)=1$ and $[A|B| C|D| E](k)=\sum_{l=1}^{L} \lambda_{l}(k)\left[A_{l}\left|B_{l}\right| C_{l}\left|D_{l}\right| E_{l}\right]$, where scheduling parameters $\lambda_{l}(k)$ 's are exactly known at the current time but unknown in future.

For above system (2), the dynamic output feedback controller is of the following form:

$$
\begin{aligned}
x_{c}(i+1 \mid k) & =A_{c}(k) x_{c}(i \mid k)+B_{c}(k) y(i \mid k), \\
u(i \mid k) & =C_{c}(k) x_{c}(i \mid k)+D_{c}(k) y(i \mid k),
\end{aligned}
$$

where $x_{c} \in \mathfrak{R}^{n_{x}}$ is the controller state; $\left\{A_{c}(k), B_{c}(k)\right\}$ are the controller gain matrices; $\left\{C_{c}(k), D_{c}(k)\right\}$ are the feedback gain matrices.

To deal with the saturated feedback control, the method in $[1,20]$ is utilized. At each time $k$, for matrices $H_{C}(k) \in$ $\mathfrak{R}^{n_{u} \times n_{x}}$ and $H_{D}(k) \in \mathfrak{R}^{n_{u} \times n_{y}}$, we define

$$
\begin{aligned}
& \mathscr{L}\left(H_{C}(k), H_{D}(k)\right)=\left\{\left[x_{c}(i \mid k), y(i \mid k)\right]\right. \\
& \quad \in \mathfrak{R}^{n_{c}+n_{y}}:\left|H_{C_{s}}(k) x_{c}(i \mid k)+H_{D_{s}}(k) y(i \mid k)\right| \\
& \left.\quad \leq 1, s \in\left\{1, \ldots, n_{u}\right\}\right\},
\end{aligned}
$$

where $H_{C_{s}}(k)$ and $H_{D_{s}}(k)$ represent the sth row of $H_{C}(k)$ and $H_{D}(k)$, respectively. $\mathscr{L}\left(H_{C}(k), H_{D}(k)\right)$ represents the region in $\mathfrak{R}^{n_{c}+n_{y}}$, where the auxiliary feedback controller $H_{C}(k) x_{c}(i \mid$ $k)+H_{D}(k) y(i \mid k)$ does not saturate.

Furthermore, denote $\mathscr{V}$ as the set of $n_{u} \times n_{u}$ diagonal matrices whose diagonal elements are either 1 or 0 . There are $2^{n_{u}}$ elements in $\mathscr{V}$. Suppose that the elements of $\mathscr{V}$ are labeled as $V_{f}, f \in\left\{1, \ldots, 2^{n_{u}}\right\}$. Denote $V_{f}^{-1}=I-V_{f}, V_{1}=I$, and $V_{2^{n_{x}}}^{-1}=I$. Clearly, $V_{f}^{-1} \in \mathscr{V}$ if $V_{f} \in \mathscr{V}$. The following lemma adopted from $[1,20]$ is utilized to represent the saturated output feedback controller by a convex hull.

Lemma 2. For any $\left[x_{c}(i \mid k), y(i \mid k)\right] \in \mathscr{L}\left(H_{C}(k), H_{D}(k)\right)$, $\forall i \geq 0$, the saturated input $\sigma[u(i \mid k)]$ can be represented by the convex hull of the actual controller $C_{c}(k) x_{c}(i \mid k)+D_{c}(k) y(i \mid$ $k)$ and the auxiliary controller $H_{C}(k) x_{c}(i \mid k)+H_{D}(k) y(i \mid k)$ as

$$
\begin{aligned}
& \sigma[u(i \mid k)] \\
& \quad \in \operatorname{Co}\left\{V_{f}\left[C_{c}(k) x_{c}(i \mid k)+D_{c}(k) y(i \mid k)\right]\right. \\
& \left.+V_{f}^{-1}\left[H_{C}(k) x_{c}(i \mid k)+H_{D}(k) y(i \mid k)\right]\right\}, \\
& \quad f \in\left\{1, \ldots, 2^{n_{u}}\right\} .
\end{aligned}
$$

By applying Lemma 2, the saturated output feedback controller, with $\left[x_{c}(i \mid k), y(i \mid k)\right] \in \mathscr{L}\left(H_{C}(k), H_{D}(k)\right), \forall i \geq$ 0 , can be expressed as

$$
\begin{aligned}
& \sigma[u(i \mid k)]=\sum_{f=1}^{2^{n_{u}}} \eta_{f}(k) \\
& \cdot\left\{V_{f}\left[C_{c}(k) x_{c}(i \mid k)+D_{c}(k) y(i \mid k)\right]\right. \\
& \left.+V_{f}^{-1}\left[H_{C}(k) x_{c}(i \mid k)+H_{D}(k) y(i \mid k)\right]\right\},
\end{aligned}
$$

where $\eta_{f}(k)$ 's are exactly known at the current time $k$ and satisfy $\sum_{f=1}^{2^{n_{u}}} \eta_{f}(k)=1$.

The above controller parameters $\left\{A_{c}(k), B_{c}(k), C_{c}(k)\right.$, $\left.D_{c}(k), H_{C}(k), H_{D}(k)\right\}$ take the parameter-dependent form as

$$
\begin{aligned}
& A_{c}(k)=\sum_{l=1}^{L} \sum_{j=1}^{L} \sum_{f=1}^{2^{n_{u}}} \lambda_{l}(k) \lambda_{j}(k) \eta_{f}(k) \bar{A}_{c}^{l j f}, \\
& B_{c}(k)=\sum_{l=1}^{L} \sum_{f=1}^{2^{n_{u}}} \lambda_{l}(k) \eta_{f}(k) \bar{B}_{c}^{l f}, \\
& C_{c}(k)=\sum_{j=1}^{L} \lambda_{j}(k) \bar{C}_{c}^{j}, \\
& D_{c}(k)=\bar{D}_{c}, \\
& H_{C}(k)=\sum_{j=1}^{L} \lambda_{j}(k) \bar{H}_{C}^{j}, \\
& H_{D}(k)=\bar{H}_{D} .
\end{aligned}
$$

Hence, the augmented closed-loop system, based on (5), (6), (9), (10), and the predictions made by system (2), is

$$
\begin{array}{r}
\tilde{x}(i+1 \mid k)=\Phi_{l j f}(i, k) \tilde{x}(i \mid k)+\Gamma_{l j f}(i, k) w(k+i), \\
\forall i \geq 0, \tilde{x}(0 \mid k)=\tilde{x}(k),
\end{array}
$$


where

$$
\begin{aligned}
& \tilde{x}=\left[x^{\mathrm{T}}, x_{c}^{\mathrm{T}}\right]^{\mathrm{T}}, \\
& \lambda_{l}(k+i) \geq 0, \\
& \sum_{i=1}^{L} \lambda_{l}(k+i)=1 \\
& \lambda_{j}(k+i) \geq 0, \\
& \sum_{j=1}^{L} \lambda_{j}(k+i)=1 \\
& \Phi_{l j f}(i, k)=\sum_{l=1}^{L} \sum_{j=1}^{L} \sum_{f=1}^{2^{n_{u}}} \lambda_{l}(k+i) \lambda_{j}(k+i) \eta_{f}(k) \Phi_{l j f}, \\
& \Gamma_{l j f}(i, k)=\sum_{l=1}^{L} \sum_{j=1}^{L} \sum_{f=1}^{2^{n_{u}}} \lambda_{l}(k+i) \lambda_{j}(k+i) \eta_{f}(k) \Gamma_{l j f}, \\
& \Phi_{l j f}=\left[\begin{array}{cc}
A_{l}+B_{l} \bar{U}_{f} C_{j} & B_{l} \bar{W}_{f}^{j} \\
\bar{B}_{c}^{l f} C_{j} & \bar{A}_{c}^{l j f}
\end{array}\right], \\
& \Gamma_{l j f}=\left[\begin{array}{c}
B_{l} \bar{U}_{f} E_{j}+D_{l} \\
\bar{B}_{c}^{l f} E_{j}
\end{array}\right], \\
& \bar{W}_{f}^{j}(k)=\left[V_{f} \bar{C}_{c}^{j}+V_{f}^{-1} \bar{H}_{C}^{j}\right], \\
& \bar{U}_{f}(k)=\left[V_{f} \bar{D}_{c}+V_{f}^{-1} \bar{H}_{D}\right] .
\end{aligned}
$$

At each time $k$, the true state $x(k)$ is unmeasurable. Hence it is necessary to use its bounds to represent the true state. Suppose at time $k$ that $x(k)$ is confined by $m(k)$-order zonotope represented by

$$
\begin{aligned}
& x(k) \in X(k)=x_{c}(k) \oplus H(k) \mathbf{B}^{m(k)}, \\
& H(k) \in \mathfrak{R}^{n_{x} \times m(k)}, \\
& m(k) \geq n_{x} .
\end{aligned}
$$

Then the true state $x(k)$ can be represented by the following convex set:

$$
x(k) \in \operatorname{Co}\left\{\chi_{1}(k), \chi_{2}(k), \ldots, \chi_{V_{X(k)}}(k)\right\},
$$

where $\chi_{r}(k), r \in\left\{1,2, \ldots, V_{X(k)}\right\}$, are the vertices of $x(k)$; $V_{X(k)}$ is the total number of vertices of zonotope $X(k)$ at time $k$.

Remark 3. The method in [9] can be applied to obtain the vertices of $X(k)$; that is, calculate $2^{m(k)}$ vertices of zonotope $X(k)$, and apply Quickhull Algorithm in [21] to remove the redundant vertices; then $V_{X(k)}$ vertices of $X(k)$ can be obtained.
Definition 4 (robust positively invariant set). For the following dynamical system,

$$
x(k+1)=T(k) x(k)+H(k) w(k),
$$

where $[T(k) H(k)] \in \bar{\Omega} \triangleq \operatorname{Co}\left\{\left[T^{l} H^{l}\right], l=\{1, \ldots, L\}\right\}$, and bounded disturbance $w(k) \in \mathbb{W}$. If for all $x(k) \in \mathcal{S}, k \geq 0$, the condition $x(k+1) \in \mathcal{S}$ holds for all $k \geq 0$, then the convex set $\mathcal{S}$ is said to be robust positively invariant set.

\section{Main Optimization Problem and Constraints Handling}

3.1. Main Optimization Problem. At each time $k$, for $\forall i \geq 0$, the following main optimization problem with the consideration of input saturation to be solved is

$$
\begin{aligned}
& \min _{\gamma, Q, \bar{A}_{c}^{l j}, \bar{B}_{c}^{l f}, \overline{\mathrm{C}}_{c}^{j}, \bar{D}_{c}, \bar{H}_{C}^{j}, \bar{H}_{D}} \max _{[A|B| C|D| E](k+i) \in \Omega, w(k+i) \in \mathbb{D}} \gamma, \\
& \text { s.t. } \quad\|\widetilde{x}(i \mid k)\|_{Q^{-1}}^{2}-\|\widetilde{x}(i+1 \mid k)\|_{Q^{-1}}^{2} \\
& \geq \frac{1}{\gamma}\left[\|y(i \mid k)\|_{Q}^{2}+\|u(i \mid k)\|_{\mathscr{R}}^{2}\right], \\
& \tilde{x}(k) \in \varepsilon_{Q^{-1}}, \\
& {\left[x_{c}(i \mid k), y(i \mid k)\right] \in \mathscr{L}\left(H_{C}(k), H_{D}(k)\right), } \\
&-\bar{\psi} \leq \Psi y(i+1 \mid k) \leq \bar{\psi},
\end{aligned}
$$

where $\gamma$ is the performance cost; (17) is the stability/optimality condition; (18) is the augmented state constraint; (19) guarantees the auxiliary feedback controller does not saturate; (20) is the output constraint; $\mathbb{Q}$ and $\mathscr{R}$ are the positive-definite weighting matrices; $Q$ satisfies the following conditions:

$$
\begin{aligned}
Q^{-1} & =M=\left[\begin{array}{ll}
M_{1} & M_{2}^{\mathrm{T}} \\
M_{2} & M_{3}
\end{array}\right], \\
Q & =\left[\begin{array}{ll}
Q_{1} & Q_{2}^{\mathrm{T}} \\
Q_{2} & Q_{3}
\end{array}\right] .
\end{aligned}
$$

Choose $M_{2}=-M_{1}$; then $Q_{2}=Q_{3}=Q_{1}-M_{1}^{-1}, M_{3}=$ $M_{1}\left(M_{1}-Q_{1}^{-1}\right)^{-1} M_{1}$, and $M_{3}-M_{1}=\left(Q_{1}-M_{1}^{-1}\right)^{-1}$.

Remark 5. The saturated output feedback controller with the parameter-dependent form is considered in problem (16)-(20), which is different from the main optimization problem in [7-9]. Furthermore, the present paper considers componentwise bounded disturbance. Hence the methods to deal with the constraints in the main optimization problem are different from those in [7-9]. 
Similar to the constraint on the auxiliary feedback controller in $[2,22,23]$, the satisfaction of (19) guarantees that the auxiliary feedback controller does not saturate such that the saturated output feedback controller can be represented by the convex hull as (9). The constraint on the actual dynamic output feedback controller is not considered in the main optimization problem. This enables us to design $\left\{\bar{C}_{c}^{j}(k), \bar{D}_{c}(k)\right\}$ with fewer restrictions. By this way, the conservatism of actual dynamic output feedback controller design can be reduced.
3.2. Constraints Handling. In the section, the following methods are utilized to deal with (17)-(20), which consider saturated output feedback controller with the parameterdependent form and componentwise bounded disturbance.

Lemma 6. Equation (17) is satisfied if there exist scalars $\{\alpha, \gamma\}$, matrices $\left\{\widehat{A}_{c}^{l j f}, \widehat{S}_{c}^{l f}, \widehat{T}_{c}^{j f}, \widehat{U}_{f}\right\}$, positive dialog matrix $R_{w} \in$ $\mathfrak{R}^{n_{w} \times n_{w}}$, and symmetric matrices $\left\{M_{1}, Q_{1}\right\}$ such that

$$
\begin{aligned}
& \Upsilon^{S}(i, k)=\sum_{l=1}^{L} \sum_{j=1}^{L} \sum_{f=1}^{2^{n_{u}}} \lambda_{l}(k+i) \lambda_{j}(k+i) \eta_{f}(k) \Upsilon_{l j f}^{S} \geq 0, \quad \forall i \geq 0 \\
& \Upsilon_{l j f}^{S}=\left[\begin{array}{ccccccc}
\alpha M_{1} & \star & \star & \star & \star & \star & \star \\
\alpha I & \alpha Q_{1} & \star & \star & \star & \star & \star \\
0 & 0 & R_{w} & \star & \star & \star & \star \\
A_{l}+B_{l} \widehat{U}_{f} C_{j} & A_{l} Q_{1}+B_{l} \widehat{T}_{c}^{j f} & B_{l} \widehat{U}_{f} E_{j}+D_{l} & Q_{1} & \star & \star & \star \\
M_{1} A_{l}+\widehat{S}_{c}^{l f} C_{j} & \widehat{A}_{c}^{l j f} & \widehat{S}_{c}^{l f} E_{j}+M_{1} D_{l} & I & M_{1} & \star & \star \\
Q^{1 / 2} C_{j} & Q^{1 / 2} C_{j} Q_{1} & Q^{1 / 2} E_{j} & 0 & 0 & \gamma I & \star \\
\mathscr{R}^{1 / 2} \widehat{U}_{f} C_{j} & \mathscr{R}^{1 / 2} \widehat{T}_{c}^{j f} & \mathscr{R}^{1 / 2} \widehat{U}_{f} E_{j} & 0 & 0 & 0 & \gamma I
\end{array}\right], \\
& \operatorname{tr}\left(R_{w}\right) \leq 1-\alpha .
\end{aligned}
$$

Furthermore, if (22) is feasible, then (10) can be parameterized as

$$
\begin{aligned}
\bar{D}_{c} & =\widehat{D}_{c}, \\
\bar{H}_{D} & =\widehat{H}_{D}, \\
\bar{W}_{f}^{j} & =\left(\widehat{T}_{c}^{j f}-\bar{U}_{f} C_{j} Q_{1}\right) Q_{2}^{-1}, \\
\bar{C}_{c}^{j} & =\bar{W}_{1}^{j}, \\
\bar{H}_{C}^{j} & =\bar{W}_{2_{x}^{n}}^{j,} \\
\bar{B}_{c}^{l f} & =M_{2}^{-\mathrm{T}}\left(\widehat{S}_{c}^{l f}-M_{1} B_{l} \bar{U}_{f}\right), \\
\bar{A}_{c}^{l j f} & =M_{2}^{-\mathrm{T}}\left(\widehat{A}_{c}^{l j f}-M_{1} A_{l} Q_{1}-M_{1} B_{l} \bar{U}_{f} C_{j} Q_{1}\right. \\
& \left.-M_{2}^{\mathrm{T}} \bar{B}_{c}^{l f} C_{j} Q_{1}-M_{1} B_{l} \bar{W}_{f}^{j} Q_{2}\right) Q_{2}^{-1} .
\end{aligned}
$$

Proof. By adopting (24), it is equivalent to

$$
\begin{aligned}
\widehat{D}_{c} & =\bar{D}_{c}, \\
\widehat{H}_{D} & =\bar{H}_{D}, \\
\widehat{U}_{f} & =\left[V_{f} \widehat{D}_{c}+V_{f}^{-1} \widehat{H}_{D}\right],
\end{aligned}
$$

$$
\begin{aligned}
\widehat{T}_{c}^{j f}= & \bar{U}_{f} C_{j} Q_{1}+\bar{W}_{f}^{j} Q_{2}, \\
\widehat{S}_{c}^{l f}= & M_{1} B_{l} \bar{U}_{f}+M_{2}^{\mathrm{T}} \bar{B}_{c}^{l f}, \\
\widehat{A}_{c}^{l j f}= & M_{1} A_{l} Q_{1}+M_{1} B_{l} \bar{U}_{f} C_{j} Q_{1}+M_{2}^{\mathrm{T}} \bar{B}_{c}^{l f} C_{j} Q_{1} \\
& +M_{1} B_{l} \bar{W}_{f}^{j} Q_{2}+M_{2}^{\mathrm{T}} \bar{A}_{c}^{l j f} Q_{2} .
\end{aligned}
$$

If $\tilde{x}(i \mid k) \in \varepsilon_{Q^{-1}}$ and $w(k+i) \in \mathbb{D}, \forall i \geq 0$, such that $\left[\Phi_{l j f}(i, k) \tilde{x}(i \quad k)+\Gamma_{l j f}(i, k) w(k+i)\right]^{\mathrm{T}} Q^{-1}\left[\Phi_{l j f}(i, k) \widetilde{x}(i \quad\right.$ $\left.k)+\Gamma_{l j f}(i, k) w(k+i)\right] \leq 1$, then, based on Definition 4 , the ellipsoidal set $\varepsilon_{\mathrm{O}^{-1}}$ is a robust positively invariant set for system (10). This condition is satisfied if there exist $\alpha \geq 0$ and a positive dialog matrix $R_{w} \in \Re^{n_{w} \times n_{w}}$ such that

$$
\begin{aligned}
1- & {\left[\Phi_{l j f}(i, k) \tilde{x}(i \mid k)+\Gamma_{l j f}(i, k) w(k+i)\right]^{\mathrm{T}} } \\
\cdot & Q^{-1}\left[\Phi_{l j f}(i, k) \tilde{x}(i \mid k)+\Gamma_{l j f}(i, k) w(k+i)\right] \\
& -\alpha\left(1-\tilde{x}(i \mid k)^{\mathrm{T}} Q^{-1} \tilde{x}(i \mid k)\right) \\
& -\left[\operatorname{tr}\left(R_{w}\right)-w^{\mathrm{T}}(k+i) R_{w} w(k+i)\right] \\
& \geq \frac{1}{\gamma}\left[\|y(i \mid k)\|_{\mathscr{Q}}^{2}+\|u(i \mid k)\|_{\mathscr{R}}^{2}\right], \quad \forall i \geq 0 .
\end{aligned}
$$


Clearly, (26) is satisfied for all $\tilde{x}(i \mid k)$ and disturbance $w(k+i)$ if the following conditions are satisfied:

$$
\begin{aligned}
& {\left[\begin{array}{rr}
\alpha Q^{-1} & 0 \\
0 & R_{w}
\end{array}\right]} \\
& -\left[\begin{array}{c}
\Phi_{l j f}^{\mathrm{T}}(i, k) \\
\Gamma_{l j f}^{\mathrm{T}}(i, k)
\end{array}\right] Q^{-1}\left[\Phi_{l j f}(i, k), \Gamma_{l j f}(i, k)\right] \\
& \geq \frac{1}{\gamma}\left[\begin{array}{c}
0 \\
E^{\mathrm{T}}
\end{array}\right] Q[C, 0, E] \\
& +\left[\begin{array}{c}
C^{\mathrm{T}} \\
C^{\mathrm{T}} \bar{U}_{f}^{\mathrm{T}} \\
\left(W_{f}^{j}\right)^{\mathrm{T}} \\
E^{\mathrm{T}} \bar{U}_{f}^{\mathrm{T}}
\end{array}\right] \mathscr{R}\left[\bar{U}_{f} C, W_{f}^{j}, \bar{U}_{f} E\right] \\
& \operatorname{tr}\left(R_{w}\right) \leq 1-\alpha .
\end{aligned}
$$

By applying the Schur complement, it is shown that (27) is guaranteed by

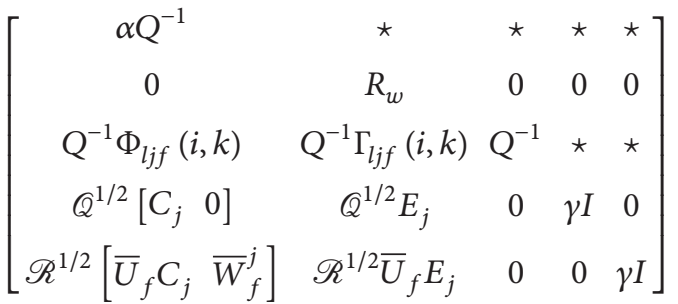

$$
\begin{aligned}
& \geq 0 \text {. }
\end{aligned}
$$

Let $T_{1}=\left[\begin{array}{ll}I & Q_{1} \\ 0 & Q_{2}\end{array}\right]$ and let $T_{2}=\left[\begin{array}{ll}I & M_{1} \\ 0 & M_{2}\end{array}\right]$. By pre- and postmultiplying the left-hand side of (29) with $\operatorname{diag}\left\{T_{1}^{\mathrm{T}}, I, T_{2}^{\mathrm{T}} \mathrm{Q}, I, I\right\}$ and $\operatorname{diag}\left\{T_{1}, I, Q T_{2}, I, I\right\}$, respectively, considering the convexity of the polytopic description of system (10) and then applying (25), one can obtain (22).

Denote the estimation error signal as $e(k) \triangleq x(k)-x_{c}(k)$. Based on the augmented state constraint in $[8,9],(18)$ holds if there exist positive-definite matrices $\left\{\bar{M}_{3}, M_{4}\right\}$ such that

$$
\begin{array}{r}
x_{c}^{\mathrm{T}}(k)\left(\bar{M}_{3}-M_{1}\right) x_{c}(k)+e^{\mathrm{T}}(k) M_{1} e(k) \leq 1, \\
{\left[\begin{array}{cc}
\bar{M}_{3} & -M_{1} \\
-M_{1} & M_{4}
\end{array}\right] \geq 0,} \\
{\left[\begin{array}{cc}
M_{1}-M_{4} & I \\
I & Q_{1}
\end{array}\right] \geq 0 .}
\end{array}
$$

The estimation error $e(k)$ can be represented by the polyhedral set; that is, $e(k) \in \mathscr{E}(k) \triangleq \operatorname{Co}\left\{\epsilon_{1}(k), \epsilon_{2}(k), \ldots, \epsilon_{V_{\mathscr{o}(k)}}(k)\right\}$, where $\epsilon_{r}(k), r \in\left\{1,2, \ldots, V_{\mathscr{E}(k)}\right\}$, are the vertices of $\mathscr{E}(k)$; $V_{\mathscr{E}(k)}$ is the total number of vertices related to $\mathscr{E}(k)$. Hence, similar to the polyhedral estimation error constraint in $[8,9]$, (30) is guaranteed by

$$
\begin{array}{r}
x_{c}(k)^{\mathrm{T}}\left(\bar{M}_{3}-M_{1}\right) x_{c}(k)+\epsilon_{j}^{\mathrm{T}}(k) M_{1} \epsilon_{j}(k) \leq 1, \\
j=1, \ldots, V_{\mathscr{E}(k)}(k) .
\end{array}
$$

Furthermore, the estimation error set $e(k)$ can also be confined by an ellipsoid; that is, $e(k) \in \varepsilon_{M_{e}(k)}$, where $M_{e}(k)$ is known at time $k$. In this case, based on the ellipsoidal estimation error set in [8], (30) is guaranteed by

$$
\begin{aligned}
x_{c}^{\mathrm{T}}(k)\left(\bar{M}_{3}-M_{1}\right) x_{c}(k) & \leq 1-\varrho, \\
M_{1} & \leq \varrho M_{e} .
\end{aligned}
$$

Lemma 7. Suppose at time $k$ that (22), (23), (30), and (31) are satisfied. If (34) is satisfied,

$$
\begin{array}{r}
\bar{\Upsilon}_{j}=\left[\begin{array}{cccr}
M_{1} & \star & \star & \star \\
I & Q_{1} & \star & \star \\
0 & 0 & R_{w} & \star \\
\sqrt{2} \xi_{s} \widehat{H}_{D} C_{j} & \sqrt{2} \xi_{s} \widehat{T}_{c}^{j f} & \sqrt{2} \xi_{s} \widehat{H}_{D} E_{j} & 1
\end{array}\right] \geq 0, \\
j=1, \ldots, L, s=1, \ldots, n_{u}, f=2^{n_{u}},
\end{array}
$$

then (19) is guaranteed.

Proof. Since (22), (23), (30), and (31) are satisfied, it can be inferred that $\tilde{x}(i \mid k) \in \varepsilon_{\mathrm{Q}^{-1}}, \forall i \geq 0$. Define $\xi_{s}$ as the sth row of the $n_{u}$-order identity matrix. Then

$$
\begin{aligned}
\max _{i \geq 0}\left|\xi_{s}\left[H_{C} x_{c}(i \mid k)+H_{D} y(i \mid k)\right]\right|^{2} \\
\quad=\max _{i \geq 0} \mid \xi_{s}\left[H_{D} C(k+i), H_{C}, H_{D} E(k+i)\right] \\
\left.\quad \cdot\left[\begin{array}{c}
\tilde{x}(i \mid k) \\
w(k+i)
\end{array}\right]\right|^{2} \\
\quad \leq \max _{i \geq 0} \| \xi_{s}\left[H_{D} C(k+i), H_{C}, H_{D} E(k+i)\right]
\end{aligned}
$$




$$
\begin{aligned}
& \cdot\left[\begin{array}{cc}
Q^{1 / 2} & 0 \\
0 & R_{w}^{-1 / 2}
\end{array}\right]\left\|^{2}\right\|\left[\begin{array}{cc}
Q^{-1 / 2} & 0 \\
0 & R_{w}^{1 / 2}
\end{array}\right]\left[\begin{array}{c}
\tilde{x}(i \mid k) \\
w(k+i)
\end{array}\right] \|^{2} \\
& \leq 2 \max _{i \geq 0} \| \xi_{s}\left[H_{D} C(k+i), H_{C}, H_{D} E(k+i)\right] \\
& \cdot\left[\begin{array}{cc}
Q^{1 / 2} & 0 \\
0 & R_{w}^{-1 / 2}
\end{array}\right] \|^{2} .
\end{aligned}
$$

If (36) is satisfied,

$$
\begin{aligned}
& 1-2 \xi_{s}\left[H_{D} C(k+i), H_{C}, H_{D} E(k+i)\right] \\
& \cdot\left[\begin{array}{cc}
Q & 0 \\
0 & R_{w}^{-1}
\end{array}\right]\left[\begin{array}{c}
{\left[H_{D} C(k+i), H_{C}\right]^{\mathrm{T}} \xi_{s}^{\mathrm{T}}} \\
E(k+i)^{\mathrm{T}} H_{D}^{\mathrm{T}} \xi_{s}^{\mathrm{T}}
\end{array}\right] \geq 0,
\end{aligned}
$$

$$
\Upsilon^{h}(i, k)=\sum_{l=1}^{L} \sum_{j=1}^{L} \sum_{f=1}^{2^{n_{u}}} \lambda_{l}(k+i) \lambda_{j}(k+i) \eta_{f}(k) \Upsilon_{l j f}^{h} \geq 0, \quad \forall i \geq 0
$$

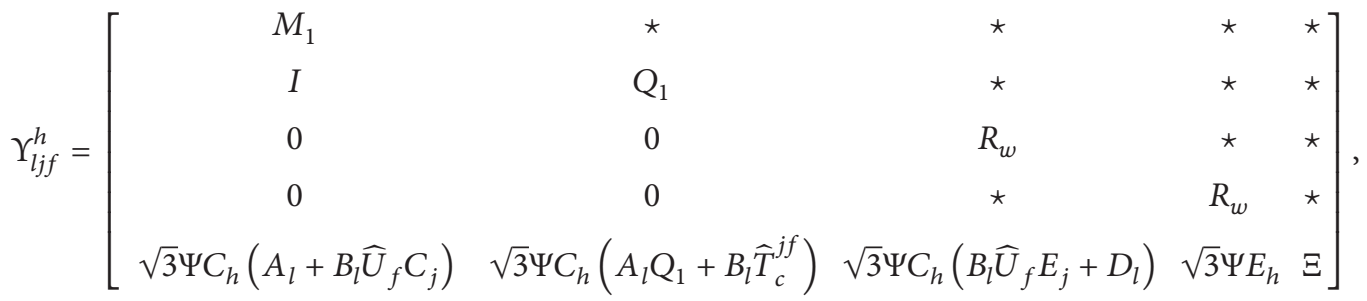

$$
\begin{aligned}
& \Xi_{s s} \leq \bar{\psi}_{s}^{2}, s \in\{1, \ldots, q\}, h \in\{1, \ldots, L\},
\end{aligned}
$$

where $\Xi_{s s}$ is the sth diagonal element of $\Xi$, then (20) is satisfied.

Proof. Define $\xi_{s}$ as the sth row of the $q$-order identity matrix. Then,

$$
\begin{aligned}
& \max _{i \geq 0}\left|\xi_{s} \Psi y(i+1 \mid k)\right|^{2}
\end{aligned}
$$

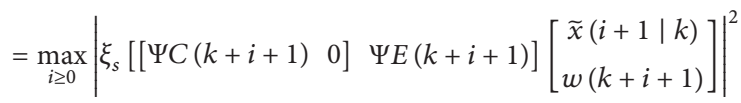

$$
\begin{aligned}
& =\max _{i \geq 0}\left|\xi_{s} \Pi_{l j f}(i+1, i, k)\left[\begin{array}{c}
\tilde{x}(i \mid k) \\
w(k+i) \\
w(k+i+1)
\end{array}\right]\right|^{2} \\
& \leq 3 \max _{i \geq 0}\left\|\xi_{s} \Pi_{l j f}(i+1, i, k)\left[\begin{array}{ccc}
Q^{1 / 2} & \star & \star \\
0 & R_{w}^{-1 / 2} & \star \\
0 & 0 & R_{w}^{-1 / 2}
\end{array}\right]\right\|^{2} \text {, }
\end{aligned}
$$

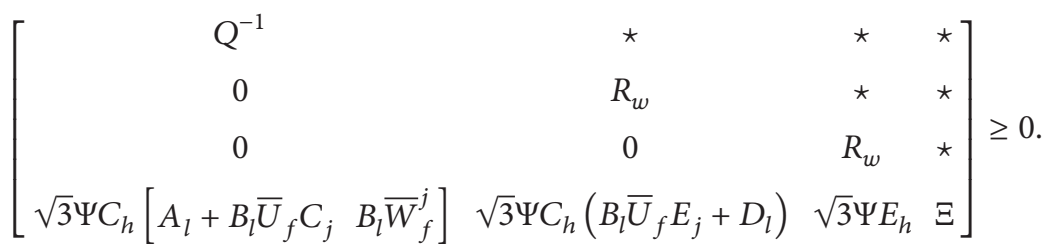
satisfying (39) such that$$
\geq 0 \text {, }
$$

then $|\Psi y(i+1 \mid k)| \leq \bar{\psi}, \forall i \geq 0$. By applying the Schur complement, it is shown that (41) is equivalent to

where $\Pi_{l j f}(i+1, i, k)=\left[[\Psi C(k+i+1) \quad 0]\left[\Phi_{l j f}(i, k) \Gamma_{l j f}(i\right.\right.$, $k)], \Psi E(k+i+1)]$. If there exists a symmetric matrix $\Xi$

$$
\Xi-3 \Pi_{l j f}(i+1, i, k)\left[\begin{array}{ccc}
Q & \star & \star \\
0 & R_{w}^{-1} & \star \\
0 & 0 & R_{w}^{-1}
\end{array}\right] \Pi_{l j f}^{\mathrm{T}}(i+1, i, k)
$$

By pre- and postmultiplying the left-hand side of (37) with $\operatorname{diag}\left\{T_{1}^{\mathrm{T}}, I, I\right\}$ and $\operatorname{diag}\left\{T_{1}, I, I\right\}$, respectively, and then apply-

Lemma 8. Suppose at time $k$ that (22), (23), (30), and (31) are satisfied. If there exists a positive-definite matrix $\{\Xi\}$ such that 
By pre- and postmultiplying the left-hand side of (42) with $\operatorname{diag}\left\{T_{1}^{\mathrm{T}}, I, I, I\right\}$ and $\operatorname{diag}\left\{T_{1}, I, I, I\right\}$, respectively, considering the convexity of the polytopic description of system (11), and then applying (25), (38) is obtained.

By applying "Proposition 2" in [24], when the complexity parameter in [24] is $n=2$, the sufficient conditions for (22) and (38) to hold are

$$
\begin{aligned}
& \Upsilon_{l l f}^{S} \geq 0, \\
& \Upsilon_{l j f}^{S}+\Upsilon_{j l f}^{S} \geq 0, \\
& \Upsilon_{l l f}^{h} \geq 0, \\
& \Upsilon_{l j f}^{h}+\Upsilon_{j l f}^{h} \geq 0, \\
& h, l, j \in\{1, \ldots, L\}, \quad l<j .
\end{aligned}
$$

With larger $n$, less conservative conditions can be obtained, but the computational burden is heavier. By applying "Proposition 2" in [24] and considering the convex representation of the input saturation, the number of LMIs incurred by (43) is $2^{n_{u}}(L+n-1) ! / n !(L-1) !=2^{n_{u}-1}(L+1) ! /(L-1) !$ and that by $(44)$ is $L\left(2^{n_{u}}(L+n-1) ! / n !(L-1) !\right)=L\left(2^{n_{u}-1}(L+1) ! /(L-1) !\right)$.

By summarizing the above derivations, it is shown that when the estimation error is represented by the polyhedral set $\mathscr{E}(k)$, problem (11)-(15) can be approximated by

$$
\begin{aligned}
\min _{\gamma, \alpha, R_{w}, Q_{1}, M_{1}, \bar{M}_{3}, M_{4}, \widehat{A}_{c}^{l j f},{ }_{B}^{l f}, \widehat{T}_{c}^{j f}, \widehat{S}_{c}^{l f}, \widehat{D}_{c}, \widehat{H}_{D}, \Xi} & \gamma, \\
\text { s.t. } & (23),(31),(32),(34),(39),(43),(44) .
\end{aligned}
$$

When the estimation error is confined by the ellipsoidal set $\varepsilon_{M_{e}(k)}$, problem (11)-(15) can be approximated by

$$
\min _{\gamma, \varrho, \alpha, R_{w}, Q_{1}, M_{1}, \bar{M}_{3}, M_{4}, \widehat{A}_{c}^{l j f}, \widehat{B}_{c}^{l f}, \widehat{T}_{c}^{j f}, \widehat{S}_{c}^{l f}, \widehat{D}_{c}, \widehat{H}_{D}, \Xi} \gamma,
$$

$$
\text { s.t. (23), (31), (32), (34), (39), (43), (44). }
$$

Problems (45) and (46) can be solved by an LMI tool for the fixed scalar $\alpha$ over $(0,1)$. When problem (45) or (46) is solved at time $k$, the controller parameters are calculated by (10) and (24). The representation of saturated output feedback controller by the parameter-dependent form of both the actual output feedback controller and the auxiliary feedback controller increases the decision variables and also the LMI conditions to describe constraints. Then the computational burden is increased (see Section 6).

3.3. The Recursive Feasibility. At each time $k$, based on Proposition 3 in [8], when problem (45) or (46) is solved, the optimal solution for problem (45) or (46) can be obtained. At time $k+1$, by choosing

$$
\begin{aligned}
& \bar{M}_{3}(k+1)=M_{1}^{*}(k)\left[M_{1}^{*}(k)-Q_{1}^{*}(k)^{-1}\right]^{-1} M_{1}^{*}(k), \\
& M_{4}(k+1)=M_{1}^{*}(k)-Q_{1}^{*}(k)^{-1}, \\
& \varrho(k+1)=1-\left\|x_{c}(k+1)\right\|_{\left[Q_{1}^{*}(k)-M_{1}^{*}(k)^{-1}\right]^{-1}} \\
& M_{e}(k+1)=M_{1}^{*}(k) \varrho(k+1)^{-1},
\end{aligned}
$$

$$
\begin{aligned}
& \left\{\gamma, \alpha, R_{w}, Q_{1}, M_{1}, \widehat{A}_{c}^{l j f}, \widehat{B}_{c}^{l f}, \widehat{T}_{c}^{j f}, \widehat{S}_{c}^{l f}, \widehat{D}_{c}, \widehat{H}_{D}, \Xi\right\}(k \\
& \quad+1) \\
& \quad=\left\{\gamma, \alpha, R_{w}, Q_{1}, M_{1}, \widehat{A}_{c}^{l j f}, \widehat{B}_{c}^{l f}, \widehat{T}_{c}^{j f}, \widehat{S}_{c}^{l f}, \widehat{D}_{c}, \widehat{H}_{D}, \Xi\right\}^{*}(k),
\end{aligned}
$$

the feasible solution for problem (46) can be constructed.

Furthermore, in problem (45) or (46), (43) guarantees that augmented state $\tilde{x}(i+1 \mid k), \forall i \geq 0$, will converge within

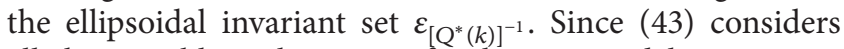
all the possible realizations of polytopic model parametric uncertainty and bounded disturbance, $\tilde{x}(1 \mid k) \in \varepsilon_{\left[Q^{*}(k)\right]^{-1}}$ leads to $\tilde{x}(k+1) \in \varepsilon_{\left[Q^{*}(k)\right]^{-1}}$; that is,

$$
\begin{aligned}
\tilde{x}^{\mathrm{T}}(k+1) M^{*}(k) \tilde{x}(k+1) \\
=x_{c}^{\mathrm{T}}(k+1)\left(M_{3}^{*}-M_{1}^{*}\right) x_{c}(k+1) \\
\quad+e^{\mathrm{T}}(k+1) M_{1}^{*} e(k+1) \leq 1 \\
=x_{c}^{\mathrm{T}}(k+1)\left[Q_{1}^{*}(k)-M_{1}^{*}(k)^{-1}\right]^{-1} x_{c}(k+1) \\
\quad+e^{\mathrm{T}}(k+1) M_{1}^{*} e(k+1) \leq 1 .
\end{aligned}
$$


Based on the selected parameters in (48), it can be obtained that $(50)$ is equivalent to

$$
e^{\mathrm{T}}(k+1) M_{e}(k+1) e(k+1) \leq 1 ;
$$

that is, based on the invariance of the augmented closed-loop system, the estimation error set at time $k+1$ is confined by an ellipsoidal set $\varepsilon_{M_{e}(k+1)}$. Note that the true estimation error must satisfy (51), which has considered all the possible realizations of polytopic model parametric uncertainty and bounded disturbance. Hence, it may be conservative for the estimation error constraint at time $k+1$. If we can obtain the bounds of the polyhedral set $\mathscr{E}(k+1)$ and satisfy $\mathscr{E}(k+$ 1) $\subset \varepsilon_{M_{e}(k+1)}$, then (32) is less conservative than (33) for the estimation error constraint at time $k+1$. Solving problem (45) at time $k+1$ can obtain better control performance than solving problem (46). If $\mathscr{E}(k+1) \not \subset \varepsilon_{M_{e}(k+1)}$, then $\mathscr{E}(k+1)$ exceeds the bounds of $\varepsilon_{M_{e}(k+1)}$. In this case, solve problem (46) at time $k+1$, and apply a central symmetric polyhedral set for outer approximating $\varepsilon_{M_{e}(k+1)}$. By this way, the estimation error set at time $k+1$ is also a polyhedral set. In the following section, we apply the zonotopic set-membership estimation approach for refreshing the polyhedral estimation error set.

\section{The Refreshment of Estimation Error Set}

At each time $k$, for quasi-LPV system (2), the model parameters are known at the current time $k$. The estimated state $x_{c}(k+$ 1 ) is calculated by (5). When the control input is calculated by (6) and implemented to system (2), then, according to (2) and the definition of estimation error, it is shown that

$$
\begin{aligned}
e(k+1)= & A(k) x(k)+B(k) \sigma[u(k)]+D(k) w(k) \\
& -x_{c}(k+1) .
\end{aligned}
$$

By applying (3), (13), and zonotopic computation properties 1 and 2 in [9], it can be obtained that

$$
\begin{aligned}
e(k+1) \in & P(k+1) \\
\triangleq & {\left[A(k) x_{c}(k)+B(k) \sigma[u(k)]-x_{c}(k+1)\right] } \\
& \oplus[A(k) H(k), D(k)] \mathbf{B}^{m(k)+n_{w}} .
\end{aligned}
$$

From (53), the estimation error set at time $k+1$ can be represented by $m(k)+n_{w}$-order zonotope $P(k+1) \triangleq p(k+$ 1) $\oplus \bar{H}(k+1) \mathbf{B}^{m(k)+n_{w}}$, where

$$
\begin{aligned}
p(k+1)= & A(k) x_{c}(k)+B(k) \sigma[u(k)] \\
& -x_{c}(k+1), \\
\bar{H}(k+1)= & {[A(k) H(k), D(k)] . }
\end{aligned}
$$

It can be obtained from (53) that, with the evolution of time, the order of zonotope $P(k+1)$ will increase. Accordingly, the generators and the vertices of zonotope $P(k+1)$ will increase, which may lead to more LMIs in the main optimization problem to describe the estimation error constraints and then increase the on-line computational burden of the main optimization problem. Hence, we impose the restriction on the order of $P(k+1)$ by $m(k)+n_{w} \leq N_{z}$. When $m(k)+$ $n_{w}>N_{z}$, Property 3 in [9] is applied to construct a loworder $N_{z}$-zonotope $\widehat{P}(k+1)=p(k+1) \oplus \widehat{H}(k+1) \mathbf{B}^{N_{z}}$ for outer approximating the high-order zonotope $P(k+1)$; that is, $P(k+1) \subseteq \widehat{P}(k+1)$, where

$$
\begin{aligned}
\widehat{H}(k+1)=\left[\begin{array}{ll}
\widehat{H}_{T}(k+1) & O(k+1)
\end{array}\right], \\
\widehat{H}(k+1) \in \mathfrak{R}^{n_{x} \times N_{z}},
\end{aligned}
$$

$\widehat{H}_{T}(k+1) \in \mathfrak{R}^{n_{x} \times\left[N_{z}-n_{x}\right]}$, and $O(k+1) \in \mathfrak{R}^{n_{x} \times n_{x}}$ are calculated according to (2) and (3) in [9], respectively. From the above derivations, at time $k+1$, the estimation error set is of one of the following forms:

$$
\begin{aligned}
& \mathscr{E}(k+1)=P(k+1), \\
& H(k+1)=\bar{H}(k+1), \\
& m(k+1)=m(k)+n_{w}, \quad \text { when } m(k)+n_{w} \leq N_{z} ; \\
& \mathscr{E}(k+1)=\widehat{P}(k+1), \\
& H(k+1)=\widehat{H}(k+1), \\
& m(k+1)=N_{z}, \quad \text { when } m(k)+n_{w}>N_{z} .
\end{aligned}
$$

When the estimation error set $\mathscr{E}(k+1)$ is obtained, it can be described by $V_{\mathscr{E}(k+1)}$ vertices; that is, $\mathscr{E}(k+1) \triangleq \operatorname{Co}\left\{\epsilon_{1}(k+\right.$ $\left.1), \epsilon_{2}(k+1), \ldots, \epsilon_{V_{\mathscr{E}(k+1)}}(k+1)\right\}$.

At each time $k$, when $\mathscr{E}(k+1)$ is obtained by (56) or (57) and $\varepsilon_{M_{e}(k+1)}$ is obtained by (48), it can be obtained that if the following problem is feasible,

$$
\begin{aligned}
& \text { find } \eta, \\
& \text { s.t. }\left[\begin{array}{cc}
\eta & \star \\
\epsilon_{r}(k+1) & M_{e}^{-1}(k+1)
\end{array}\right] \geq 0, \\
& 0 \leq \eta \leq 1, r \in\left\{1,2 \ldots, V_{\mathscr{E}(k+1)}\right\},
\end{aligned}
$$

then it will satisfy the fact that $\mathscr{E}(k+1) \subset \varepsilon_{M_{e}(k+1)}$. If problem (58) is infeasible, then $\mathscr{E}(k+1) \not \subset \varepsilon_{M_{e}(k+1)}$. For this case, we apply the following method in [8] for outer approximation of $\varepsilon_{M_{e}(k+1)}$ by a polyhedral set.

Define the polyhedral set $\widetilde{\mathscr{P}}_{e}^{0}(k+1) \triangleq\{e \mid-\bar{e} \leq$ $\left.T M_{e}^{1 / 2}(k+1) e \leq \bar{e}\right\}$, where $T=\left[T_{1}^{\mathrm{T}}, T_{2}^{\mathrm{T}}, \ldots, T_{p}^{\mathrm{T}}\right]^{\mathrm{T}} \in \Re^{p \times n_{x}}$ is a transformation matrix and $\bar{e}=\left[\bar{e}_{1}, \bar{e}_{2}, \ldots, \bar{e}_{p}\right]^{\mathrm{T}} ; \bar{e}_{j}>0$, $j=1, \ldots, p$. Both $T$ and $\bar{e}$ are off-line prespecified. Let $g_{i}^{\prime}=\bar{e}_{i}^{-1}\left(T_{i} T_{i}^{\mathrm{T}}\right)^{1 / 2}, j=1, \ldots, p$. Then the set $\widetilde{\mathscr{P}}_{e}(k+$ $1) \triangleq \operatorname{diag}\left\{g_{1}^{\prime}, g_{2}^{\prime}, \ldots, g_{p}^{\prime}\right\} \widetilde{\mathscr{P}}_{e}^{0}(k+1)$ can perform the outer approximation of the set $\varepsilon_{M_{e}(k+1)}$. In [8], $\widetilde{\mathscr{P}}_{e}(k+1)$ is a central symmetric polyhedral set with the center of origin. Hence, it can be represented by a zonotope. By applying the zonotopic computation method in PnPMPC Toolbox [25], we denote it as $\widetilde{P}(k+1)=0 \oplus \widetilde{H}(k+1) \mathbf{B}^{N_{e}}$. 
Remark 9. In [7-9], the main optimization problem considers an ellipsoidal bounded disturbance, while in the refreshment of estimation error set, the outer approximation of the ellipsoidal bounded disturbance by the polyhedral set is utilized, which may introduce conservatism. However, the main optimization problem and the refreshment of estimation error set in the present paper consider the componentwise bounded disturbance, which is represented by the zonotope (see (3)). Hence, the outer approximation of bounded disturbance is not involved in the refreshment of estimation error set.

\section{The Overall Solution to the Saturated Dynamic OFRMPC}

The overall solution includes solving problem (45) or (46) and calculating the controller parameters by (10) and (24) then refreshing the estimation error set. We summarize the overall algorithm as follows.

Algorithm 10 (the overall solution to the saturated dynamic OFRMPC). Prespecify a scalar $\alpha \in(0,1), T$, and $\bar{e}$. Choose a feasible $x_{c}(0)$, and $x(0) \in x_{c}(0) \oplus H(0) \mathbf{B}^{m(0)}$, where $H(0) \in$ $\mathfrak{R}^{n_{x} \times m(0)}$. Select the maximal order of zonotope related to the estimation error set $\mathscr{E}(k)$ as $N_{z}$. Let flag $=1$. At each time $k \geq 0$, perform the following steps:

(1) If flag $=1$, then solve problem (45); else solve problem (46).

(2) Calculate the controller parameters by (10) and (24). Implement the control input by (6). Obtain $x_{c}(k+1)$ and set $\varepsilon_{M_{e}(k+1)}$ by (5) and (48), respectively.

(3) Calculate $\mathscr{E}(k+1)$ by (53). If $m(k)+n_{w} \leq N_{z}$, then $\mathscr{E}(k+1)=P(k+1) ;$ else $\mathscr{E}(k+1)=\widehat{P}(k+1)$.

(4) If problem (58) is feasible, then let flag $=1$; else let flag $=0$, perform outer approximation of $\varepsilon_{M_{e}(k+1)}$ by $\widetilde{\mathscr{P}}_{e}(k+1)$, represent it by zonotope $\widetilde{P}(k+1)$, and let $\mathscr{E}(k+1)=\widetilde{P}(k+1)$.

Theorem 11. For uncertain system (2), suppose that $w(k) \in$ $\mathbb{D}$ for all $k \geq 0$ and $x(0) \in x_{c}(0) \oplus H(0) \mathbf{B}^{m(0)}$. By applying Algorithm 10, if problem (45) is feasible at time $k=0$, then the recursive feasibility is guaranteed. The augmented state $\tilde{x}(k)$ will converge to a neighborhood of $\tilde{x}=0$; the input and the output constraints are satisfied for all $k \geq 0$.

Proof. As mentioned in Section 3.3, based on Proposition 3 in [8], when problem (45) or (46) is solved at time $k$, the optimal solution is $\left\{\gamma, \alpha, R_{w}, Q_{1}, M_{1}, \bar{M}_{3}, M_{4}, \widehat{A}_{c}^{l j f}, \widehat{B}_{c}^{l f}, \widehat{T}_{c}^{\text {jf }}\right.$, $\left.\widehat{S}_{c}^{l f}, \widehat{D}_{c}, \widehat{H}_{D}, \Xi\right\}^{*}(k)$ or $\left\{\gamma, \alpha, \varrho, R_{w}, Q_{1}, M_{1}, \bar{M}_{3}, M_{4}, \widehat{A}_{c}^{l j f}, \widehat{B}_{c}^{l f}\right.$, $\left.\widehat{T}_{c}^{j f}, \widehat{S}_{c}^{l f}, \widehat{D}_{c}, \widehat{H}_{D}, \Xi\right\}^{*}(k)$. Based on (47), (48), and (49), the feasible solution for problem (46) at time $k+1$ can be obtained. Hence, if problem (45) is feasible at time $k=0$, then, at time $k \geq 1$, consider the following two cases:

(1) If problem (58) is feasible, then let $\mathscr{E}(k+1) \subset \varepsilon_{M_{e}(k+1)}$, and solve problem (45).
(2) If problem (58) is infeasible, then let $\mathscr{E}(k+1) \not \subset$ $\varepsilon_{M_{e}(k+1)}$, solve problem $(46)$, and let $\mathscr{E}(k+1)=\widetilde{P}(k+$ $1)$.

For the above two cases, when problem (45) or (46) is solved at time $k+1$, it can guarantee that, in the main optimization problem, $e(k+1) \in \varepsilon_{M_{e}(k+1)}$ is always satisfied. Hence, based on the invariance of the augmented closed-loop system, the augmented state constraint at time $k+1$ is satisfied. By further choosing the feasible solution as (47) and (49), the recursive feasibility is guaranteed. In problem (45) or (46), (43) guarantees that

$$
\begin{aligned}
\alpha\|\tilde{x}(i \mid k)\|_{Q^{-1}}^{2}+w^{\mathrm{T}}(k+i) R_{w} w(k+i) & \\
& -\|\tilde{x}(i+1 \mid k)\|_{Q^{-1}}^{2} \\
\geq & \frac{1}{\gamma(k)}\left[\|y(i \mid k)\|_{\mathscr{Q}}^{2}+\|u(i \mid k)\|_{\mathscr{R}}^{2}\right], \quad \forall i \geq 0 .
\end{aligned}
$$

By applying the definition of bounded disturbance and (28), the following condition is satisfied:

$$
\begin{aligned}
& \alpha\|\tilde{x}(i \mid k)\|_{Q^{-1}}^{2}+1-\alpha-\|\tilde{x}(i+1 \mid k)\|_{Q^{-1}}^{2} \\
& \quad \geq \frac{1}{\gamma(k)}\left[\|y(i \mid k)\|_{\mathscr{Q}}^{2}+\|u(i \mid k)\|_{\mathscr{R}}^{2}\right], \quad \forall i \geq 0 .
\end{aligned}
$$

By summing (60) from $i=0$ to $i=\infty$ and applying $\| \widetilde{x}(i \mid$ $k) \|_{Q^{-1}}^{2} \leq 1$, it achieves

$$
J_{\infty}(k)=\sum_{i=0}^{\infty}\left[\|y(i \mid k)\|_{\mathscr{Q}}^{2}+\|u(i \mid k)\|_{\mathscr{R}}^{2}\right] \leq \gamma(k) .
$$

Hence, at each time $k, \gamma(k)$ is a feasible bound of performance cost at time $k$. By reoptimization of problem (45) or (46) at time $k+1$, it must result in $\gamma^{*}(k+1) \leq \gamma^{*}(k)$. By induction, $\gamma^{*}(k)$ will not increase with time $k$.

Furthermore, consider the following uncorrupt system (i.e., the system is not corrupted by disturbance):

$$
\begin{aligned}
& \tilde{x}_{u}(i+1 \mid k)= \Phi(i, k) \tilde{x}_{u}(i \mid k), \\
& \tilde{x}_{u}(0 \mid k)=\tilde{x}(k), \\
& u_{u}(i \mid k)= F_{x}(i \mid k) x_{c, u}(i \mid k) \\
&+F_{y}(i \mid k) y_{u}(i \mid k), \\
& y_{u}(i \mid k)= C(k+i) x_{u}(i \mid k), \quad \forall i \geq 0 .
\end{aligned}
$$

Equations (23) and (43) also guarantee

$$
\begin{aligned}
& \left\|\widetilde{x}_{u}(i \mid k)\right\|_{Q^{-1}}^{2}-\left\|\widetilde{x}_{u}(i+1 \mid k)\right\|_{Q^{-1}}^{2} \\
& \quad \geq \frac{1}{\gamma(k)}\left[\left\|y_{u}(i \mid k)\right\|_{\mathscr{Q}}^{2}+\left\|u_{u}(i \mid k)\right\|_{\mathscr{R}}^{2}\right], \quad \forall i \geq 0 .
\end{aligned}
$$

By summing (63) from $i=0$ to $i=\infty$ and then applying (62), it achieves

$$
J_{\infty}(k)=\sum_{i=0}^{\infty}\left[\left\|y_{u}(i \mid k)\right\|_{\mathscr{Q}}^{2}+\left\|u_{u}(i \mid k)\right\|_{\mathscr{R}}^{2}\right] \leq \gamma(k) .
$$




$$
\begin{aligned}
\lambda_{1} & =\frac{1}{2} \frac{\varphi_{1}(y)-\varphi_{1}(-\bar{\psi})}{\varphi_{1}(\bar{\psi})-\varphi_{1}(-\bar{\psi})}, \\
\lambda_{2} & =\frac{1}{2} \frac{\varphi_{1}(\bar{\psi})-\varphi_{1}(y)}{\varphi_{1}(\bar{\psi})-\varphi_{1}(-\bar{\psi})}, \\
\varphi_{1}(y) & =7.2 \times 10^{10} e^{-8750 /(y+350)}, \\
\lambda_{3} & =\frac{1}{2} \frac{\varphi_{2}(y)-\varphi_{2}(-\bar{\psi})}{\varphi_{2}(\bar{\psi})-\varphi_{2}(-\bar{\psi})}, \\
\lambda_{4} & =\frac{1}{2} \frac{\varphi_{2}(\bar{\psi})-\varphi_{2}(y)}{\varphi_{2}(\bar{\psi})-\varphi_{2}(-\bar{\psi})}, \\
\varphi_{2}(y) & =3.6 \times \frac{10^{10}\left(e^{-8750 /(y+350)}-e^{-8750 / 350}\right)}{y} .
\end{aligned}
$$

Furthermore, based on Lemma 2 in [20], we choose $\eta_{1}(k)=$ 1 if $C_{c}(k) x_{c}(k)+D_{c}(k) y(k)=H_{C}(k) x_{c}(k)+H_{D}(k) y(k)$; else $\eta_{1}(k)=\left(\sigma\left[C_{c}(k) x_{c}(k)+D_{c}(k) y(k)\right]-\left[H_{C}(k) x_{c}(k)+\right.\right.$ $\left.\left.H_{D}(k) y(k)\right]\right) /\left(\left[C_{c}(k)-H_{C}(k)\right] x_{c}(k)+\left[D_{c}(k)-H_{D}(k)\right] y(k)\right)$; $\eta_{2}(k)=1-\eta_{1}(k)$.

For comparing Algorithm 10 with Algorithm 8 in [9] and Algorithm 1 in [8], we choose $x_{c}(0)=[0.15,3]^{\mathrm{T}}, x(0)=$ $[0.27,4.2]^{\mathrm{T}}, \alpha=0.999$, and $\mathscr{Q}=25, \mathscr{R}=1$. The initial state $x(0) \in x_{c}(0) \oplus H(0) \mathbf{B}^{m(0)}$, where $H(0)=\operatorname{diag}\{0.12,1.2\}$; $m(0)=2$. The disturbance sequence satisfies $w \in \mathfrak{R}^{2}\left(n_{w}=\right.$ 2 ), where $\left|w_{j}\right| \leq 1, j \in\{1,2\}$, are randomly generated from the interval $[-1,1]$, which can be represented by a zonotope $\mathbb{D}=0 \oplus \mathbf{B}^{2}$. For Algorithm 8 in [9] and Algorithm 1 in [8], we choose $\alpha=0.001$ and $P_{w}=\operatorname{diag}\{0.5,0.5\}$; that is, $\varepsilon_{P_{w}} \subset \mathbb{D}$ (set $\mathbb{D}$ in the present paper is the outer approximation of bounded disturbance $\varepsilon_{P_{w}}$ in $\left.[8,9]\right)$. For Algorithm 1 in [8] and Algorithm 10, we select $\bar{e}=[0.1697,0.24,1.697,2.4]^{\mathrm{T}}$, $T=\left[\begin{array}{cccc}0.1697 & 0.1697 & 0 & -1.697 \\ 0 & 0.1697 & 1.697 & 1.697\end{array}\right]^{\mathrm{T}}$. Furthermore, for Algorithm 1 in [8], we let $M_{e}(0)=\operatorname{diag}\left\{0.5 /(0.12)^{2}, 0.5 /(1.2)^{2}\right\} ; \widehat{p}=8$. For Algorithm 10 and Algorithm 8 in [9], we restrict $N_{z}=4$ $\left(V_{\mathscr{E}(k)} \leq 8\right)$.

The simulation results are shown in Figures 1 and 2. Figure 1 compares the trajectories of the augmented closedloop system. The control input signals shown in Figure 2 illustrate that the input constraints are satisfied. The time spent on the simulations is $124.26 \mathrm{~s}$ (41.13 s and $42.58 \mathrm{~s}$ ) for Algorithm 10 (Algorithm 8 in [9] and Algorithm 1 in [8]). From the compared algorithms, Algorithm 10 in the present paper has the best control performance, but the computational burden is increased. The LMI Toolbox of Matlab 7.14 and zonotopic computation method in PnPMPC Toolbox [25] (AMD Phenom II Processor 2.70 GHz, $8 \mathrm{G}$ Memory) are utilized for the simulations.

\section{Conclusions}

This paper considers a synthesis approach of dynamical OFRMPC for the quasi-LPV system with input saturation. The saturated control input is represented by a convex hull

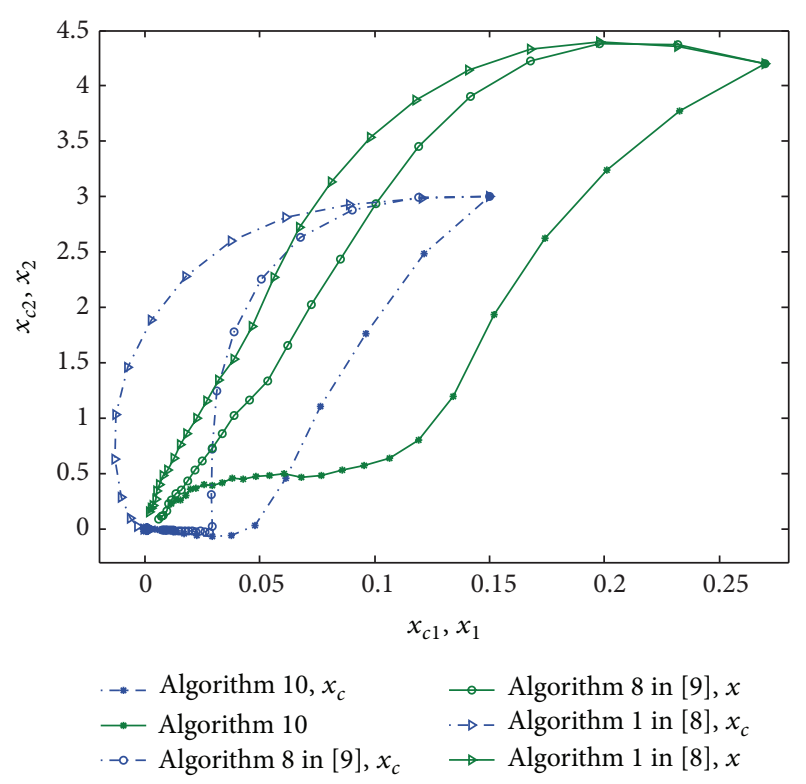

FIGURE 1: The trajectories of the augmented closed-loop system.

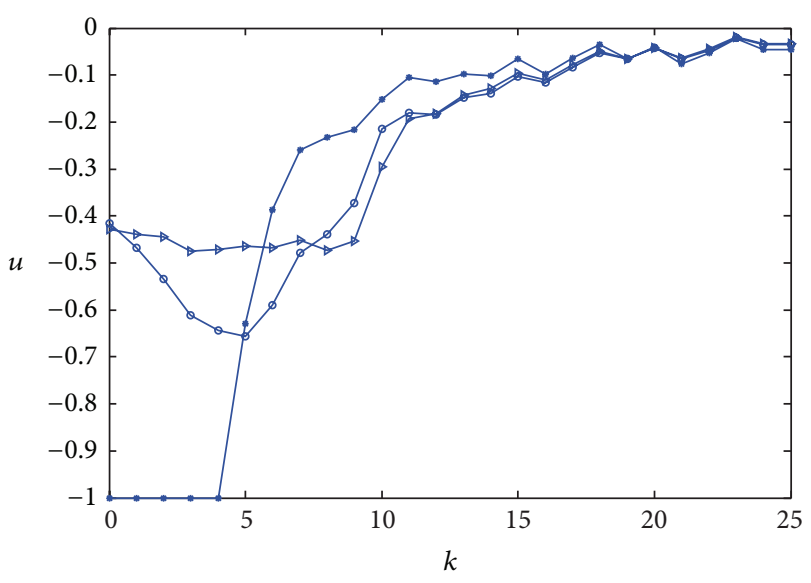

$\rightarrow$ Algorithm $10 \rightarrow$ Algorithm 8 in [9] $\rightarrow$ Algorithm 1 in [8]

FIgURE 2: The control input signals.

involving the parameter-dependent form of the actual output feedback controller and the introduced auxiliary controller. The main optimization problem is solved as convex optimization and reduces the conservatism of the dynamic output feedback controller design. The refreshment of estimation error set not only obtains the bounds of the estimation error set at the next sampling time, but also guarantees the recursive feasibility of the optimization problem. Compared with the previous results, the control performance is improved at the cost of higher computational burden.

\section{Competing Interests}

The authors declare that they have no competing interests. 


\section{Acknowledgments}

This work is supported by the National Natural Science Foundation of China (NSFC, 61403297) and the Fundamental Research Funds for the Central Universities (JB140417).

\section{References}

[1] Q. Zheng and F. Wu, "Output feedback control of saturated discrete-time linear systems using parameter-dependent Lyapunov functions," Systems \& Control Letters, vol. 57, no. 11, pp. 896-903, 2008.

[2] Y. Cao and Z. Lin, "Min-max MPC algorithm for LPV systems subject to input saturation," IEE Proceedings-Control Theory and Applications, vol. 152, no. 3, pp. 266-272, 2005.

[3] M. V. Kothare, V. Balakrishnan, and M. Morari, "Robust constrained model predictive control using linear matrix inequalities," Automatica, vol. 32, no. 10, pp. 1361-1379, 1996.

[4] Y. Lu and Y. Arkun, "Quasi-min-max MPC algorithms for LPV systems," Automatica, vol. 36, no. 4, pp. 527-540, 2000.

[5] A. Casavola, D. Famularo, and G. Franze, "A feedback min-max MPC algorithm for LPV systems subject to bounded rates of change of parameters," IEEE Transactions on Automatic Control, vol. 47, no. 7, pp. 1147-1153, 2002.

[6] D. Li and Y. Xi, "The feedback robust MPC for LPV systems with bounded rates of parameter changes," IEEE Transactions on Automatic Control, vol. 55, no. 2, pp. 503-507, 2010.

[7] B. Ding, "Constrained robust model predictive control via parameter-dependent dynamic output feedback," Automatica, vol. 46, no. 9, pp. 1517-1523, 2010.

[8] B. Ding, X. Ping, and H. Pan, "On dynamic output feedback robust MPC for constrained quasi-LPV systems," International Journal of Control, vol. 86, no. 12, pp. 2215-2227, 2013.

[9] X. Ping and N. Sun, "Dynamic output feedback robust model predictive control via zonotopic set-membership estimation for constrained quasi-LPV systems," Journal of Applied Mathematics, vol. 2015, Article ID 875850, 12 pages, 2015.

[10] D. Q. Mayne, J. B. Rawlings, C. V. Rao, and P. O. Scokaert, "Constrained model predictive control: stability and optimality," Automatica, vol. 36, no. 6, pp. 789-814, 2000.

[11] D. Piga, P. Cox, R. Tóth, and V. Laurain, "LPV system identification under noise corrupted scheduling and output signal observations," Automatica, vol. 53, pp. 329-338, 2015.

[12] X. Li and F. Zhu, "Simultaneous actuator and sensor fault estimation for descriptor LPVsystem based on $H_{\infty}$ reducedorder observer," Optimal Control Applications and Methods, 2015.

[13] D. Efimov, T. Raïssi, W. Perruquetti, and A. Zolghadri, "Design of interval observers for estimation and stabilization of discretetime LPV systems," IMA Journal of Mathematical Control and Information, Article ID dnv023, 2015.

[14] J. Mohammadpour and C. W. Scherer, Control of Linear Parameter Varying Systems with Applications, Springer Science \& Business Media, 2012.

[15] P. Mhaskar, "Robust model predictive control design for faulttolerant control of process systems," Industrial and Engineering Chemistry Research, vol. 45, no. 25, pp. 8565-8574, 2006.

[16] M. Mahmood, R. Gandhi, and P. Mhaskar, "Safe-parking of nonlinear process systems: handling uncertainty and unavailability of measurements," Chemical Engineering Science, vol. 63, no. 22, pp. 5434-5446, 2008.
[17] S. Yu, C. Böhm, H. Chen, and F. Allgöwer, "Model predictive control of constrained LPV systems," International Journal of Control, vol. 85, no. 6, pp. 671-683, 2012.

[18] B. Ding and X. Ping, "Output feedback predictive control with one free control move for nonlinear systems represented by a takagi-sugeno model," IEEE Transactions on Fuzzy Systems, vol. 22, no. 2, pp. 249-263, 2014.

[19] W. Guan and G.-H. Yang, "Analysis and design of output feedback control systems in the presence of actuator saturation," in Proceedings of the American Control Conference (ACC '09), pp. 2564-2568, St. Louis, Mo, USA, June 2009.

[20] F. Wu, Z. Lin, and Q. Zheng, "Output feedback stabilization of linear systems with actuator saturation," IEEE Transactions on Automatic Control, vol. 52, no. 1, pp. 122-128, 2007.

[21] C. B. Barber, D. P. Dobkin, and H. Huhdanpaa, "The quickhull algorithm for convex hulls," ACM Transactions on Mathematical Software, vol. 22, no. 4, pp. 469-483, 1996.

[22] H. Huang, D. Li, Z. Lin, and Y. Xi, "An improved robust model predictive control design in the presence of actuator saturation," Automatica, vol. 47, no. 4, pp. 861-864, 2011.

[23] D. Li, Y. Xi, and F. Gao, "Synthesis of dynamic output feedback RMPC with saturated inputs," Automatica, vol. 49, no. 4, pp. 949-954, 2013.

[24] A. Sala and C. Ariño, "Asymptotically necessary and sufficient conditions for stability and performance in fuzzy control: applications of Polya's theorem," Fuzzy Sets and Systems, vol. 158, no. 24, pp. 2671-2686, 2007.

[25] S. Riverso, A. Battocchio, and G. Ferrari-Trecate, PnPMPC: A Toolbox for Matlab, 2012, http://sisdin.unipv.it/pnpmpc/ pnpmpc.php.

[26] P. Gahinet, A. Nemirovski, A. J. Laub, and M. Chilali, LMI Control Toolbox for Use with MATLAB, User's Guide, The MathWorks, Natick, Mass, USA, 1995. 


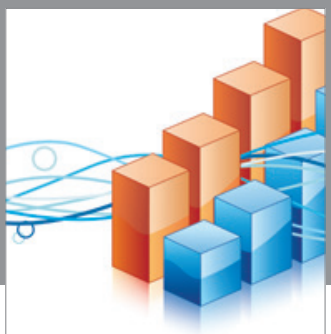

Advances in

Operations Research

vatem alat4

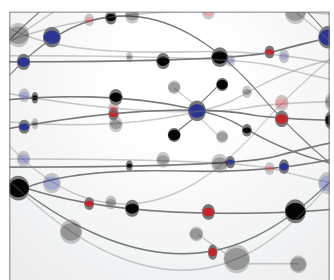

\section{The Scientific} World Journal
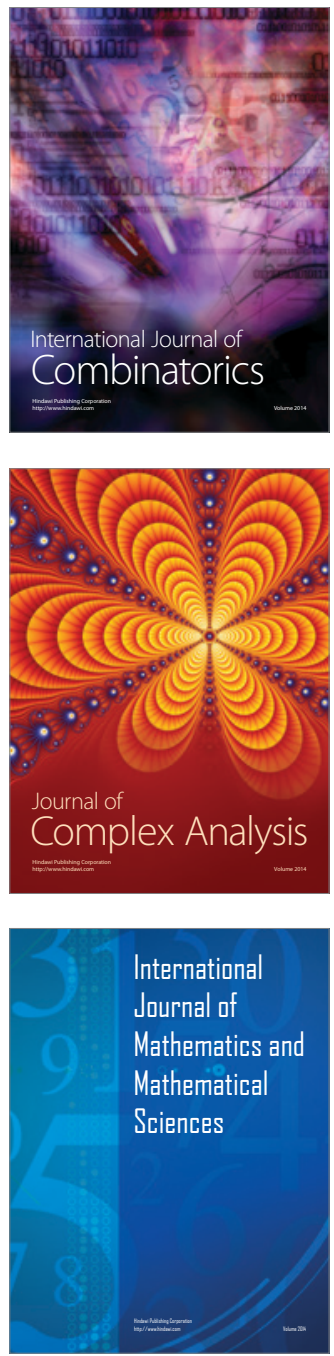
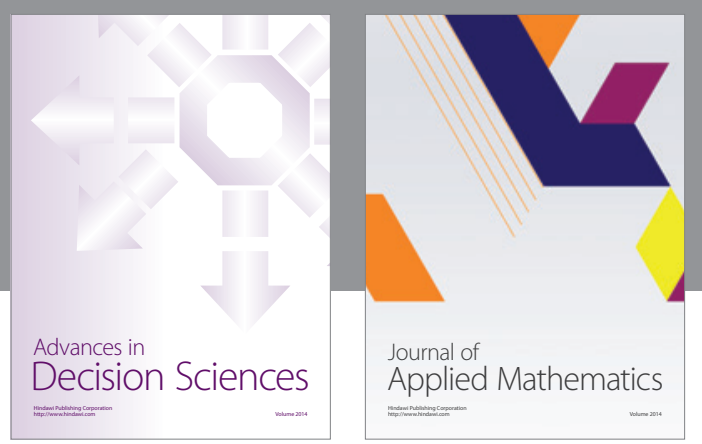

Algebra

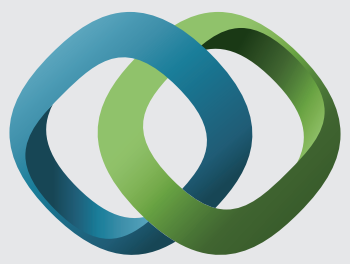

\section{Hindawi}

Submit your manuscripts at

http://www.hindawi.com
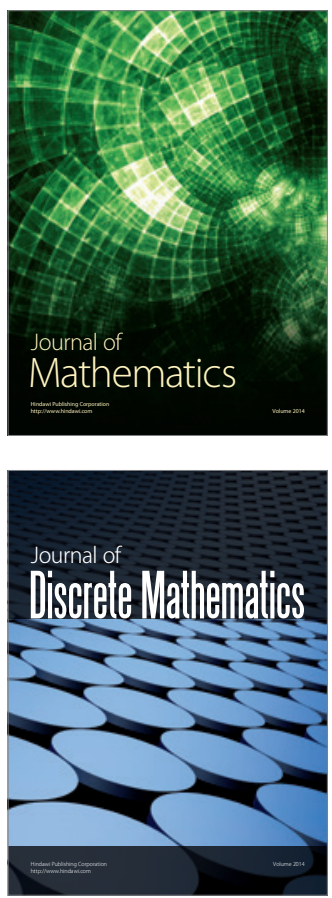

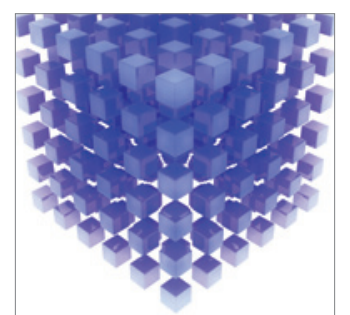

Mathematical Problems in Engineering
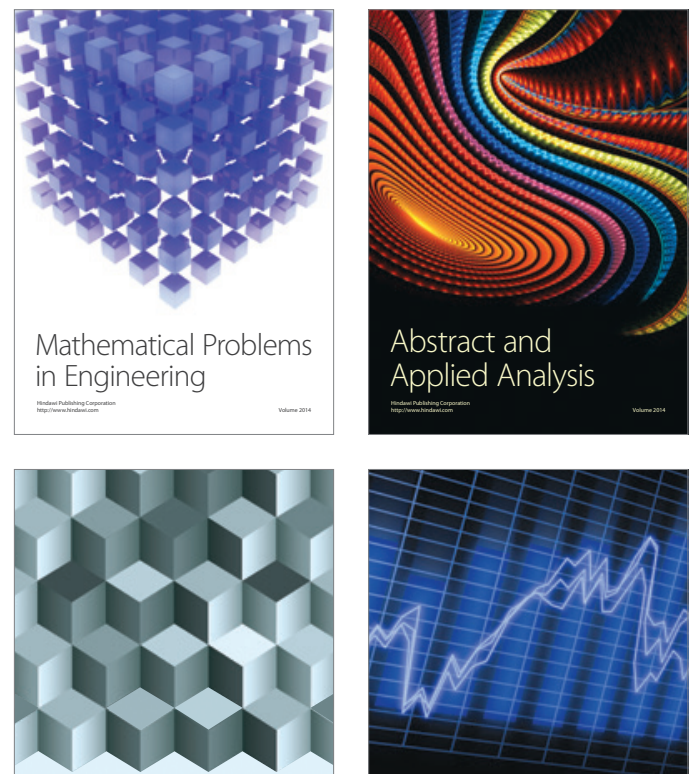

Journal of

Function Spaces

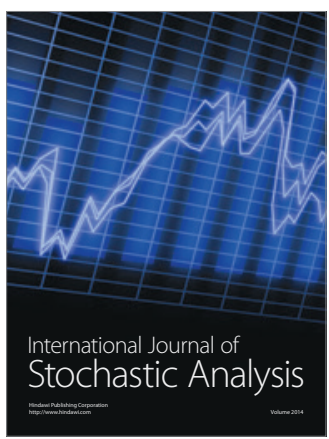

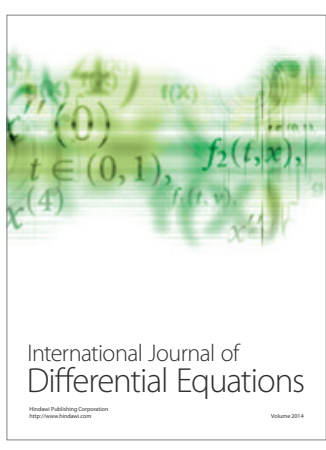
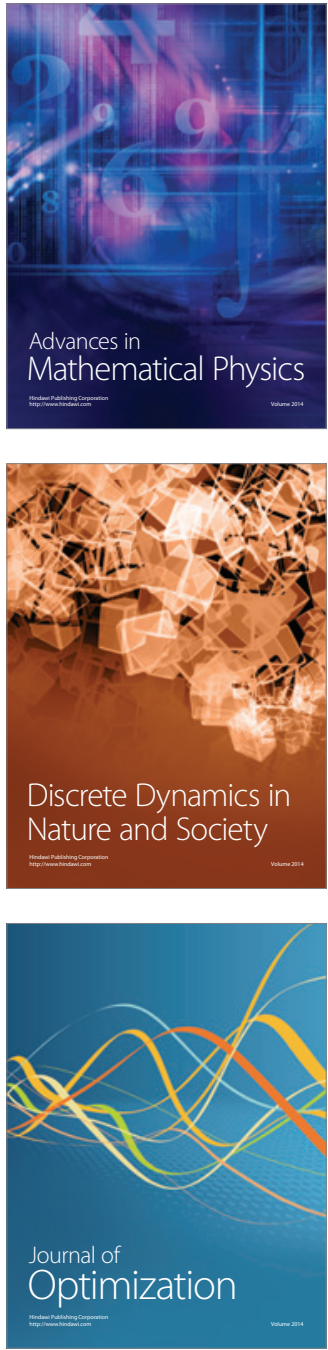